\title{
Theories relating baryon asymmetry and dark matter
}

\author{
Sofiane M. Boucenna ${ }^{1}$ and Stefano Morisi ${ }^{2 *}$ \\ 1 Instituto de Física Corpuscular, CSIC-Universitat de València, Valencia, Spain \\ ${ }^{2}$ Institut für Theoretische Physik und Astrophysik, Universität Würzburg, Würzburg, Germany
}

\section{Edited by:}

Nicolao Fornengo, University of Torino, Italy

\section{Reviewed by:}

Francesco Sannino, University of Southern Denmark, Denmark

Jihn E. Kim, Kyung Hee University,

South Korea

\section{*Correspondence:}

Stefano Morisi, Institut für

Theoretische Physik und Astrophysik,

Universität Würzburg, Campus Hubland

North, Emil-Hilb-Weg 22, 97074

Würzburg, Germany

e-mail: stefano.morisi@gmail.com
The nature of dark matter and the origin of the baryon asymmetry are two of the deepest mysteries of modern particle physics. In the absence of hints regarding a possible solution to these mysteries, many approaches have been developed to tackle them simultaneously leading to very diverse and rich models. We give a short review where we describe the general features of some of these models and an overview on the general problem. We also propose a diagrammatic notation to label the different models.

\footnotetext{
Keywords: dark matterWIMP, baryon asymmetry, asymmetric dark matter, electroweak phase transition, leptogenesis
}

\section{INTRODUCTION}

The latest results on the cosmological parameters [1] reveal that only $4.9 \%$ of the content of the Universe is in the form of baryonic matter whereas $26.8 \%$ is constituted by dark matter. The rest is accounted for by the mysterious dark energy. If we focus on the matter front then two disturbing questions are readily asked: What is the nature of dark matter? and why is its density so close to the baryonic matter density, i.e., $\Omega_{D M} \sim 5 \Omega_{B}$ ?

Moreover, the above-mentioned visible matter density does not include anti-baryons i.e., the visible universe is asymmetric with an initial excess of baryons over anti-baryons parametrized by $\eta(b)=\left(n_{b}-n_{\bar{b}}\right) / s \sim 10^{-10}$, where $n$ denotes the number density and $s$ the entropy density. Therefore, another fundamental question is what is the origin of the observed baryon asymmetry of the universe (BAU)?

This puts finding the nature of DM and the mechanism behind baryogenesis at the top of the agenda of modern physics ${ }^{1}$. While the solutions to these two problems might well be unrelated to each other, it is nevertheless tempting to assume the new physics to be minimal and unifying enough so that it solves both of them with the same ingredients. Moreover, if we discard simple numerical coincidence as an explanation to the intriguing closeness of matter densities, we are left with the task to construct theories relating them or unifying their genesis.

Indeed, numerous models have been proposed in the recent years to achieve this end. Broadly speaking, there are three approaches that are followed to relate dark matter to baryons. The first idea is that there is a sector connecting DM and baryons in the early universe. The connecting sector acts either as a parent sector, generating DM and baryons through decay for instance, or as a mediator mechanism transferring the asymmetry from the dark to the baryonic sector or vice versa. Asymmetric DM models (see below) used this approach extensively. The second approach uses the DM sector as an auxiliary to a successful

\footnotetext{
${ }^{1}$ For reviews on DM we refer the reader to $[2,3]$ and for baryogenesis to $[4,5]$.
}

baryogenesis scenario. The strength of the phase transition in electroweak baryogenesis may for instance be enhanced by the presence of DM. The third approach uses the thermal WIMP paradigm as a framework to relate the abundances.

The purpose of this mini-review is to provide a succinct yet global picture on these models focusing on the key concepts and ingredients that are used in each reviewed model and on the predictions that are made. While there are some similarities between these models, it is difficult to classify them in a consistent and easy way. Instead we opt for a diagrammatic approach Figure 1 and we review models that follow the main roads of the schematic. It is not our goal to be exhaustive with the references and we will refer to more systematic reviews when possible.

From the baryogenesis side we know that any mechanism that satisfies the three Sakharov condition[6]: B violation, C and CP violation and departure from thermal equilibrium can lead to a successful BAU. Whereas from the cold dark matter side we can generally speak of three classes of candidates: weakly interacting massive particles (WIMPs), asymmetric dark matter (ADM) and non-thermal dark matter (NTDM) ${ }^{2}$.

In principle, we can organize the paper in terms of either one of these categories, we chose however to focus on the DM nature.

The paper is organized as follows. In section 2 we review models relating DM to the baryon asymmetry while preserving the WIMP miracle. Section 3 is devoted to ADM models, where we will review different mechanisms and highlight the key concepts that are needed to construct them. In section 4 we quickly mention the possibility of non-thermal DM. Finally we summarize the different models and the roads taken in Table 1. To simplify the understanding of the different models, we will specify in the text (in bold face) whenever it is helpful and in the table the path that is followed in the schematic. We will use the following convention: $\mathrm{A} *$ denotes the stage in the diagram where a new

${ }^{2}$ Where we include any non-thermally produced DM that does not fall in the ADM case. 
asymmetry appears while a bar on the top means that the direction of the arrow is flipped. We will also use the letter $T$ to refer to a thermalization stage.

\section{WIMP DARK MATTER MODELS}

It has been noted that relic particles from a thermal bath provide in a miraculous way the correct relic density of DM. Indeed, the number density of dark particles in the primordial thermal bath is frozen-out when the expansion rate drops below the rate of the dark matter interactions. The abundance of the relic particle scales then as:

$$
\Omega_{\mathrm{CDM}} h^{2} \simeq \frac{0.3 \times 10^{-26} \mathrm{~cm}^{3} \mathrm{~s}^{-1}}{\langle\sigma v\rangle_{\text {f.o. }}},
$$

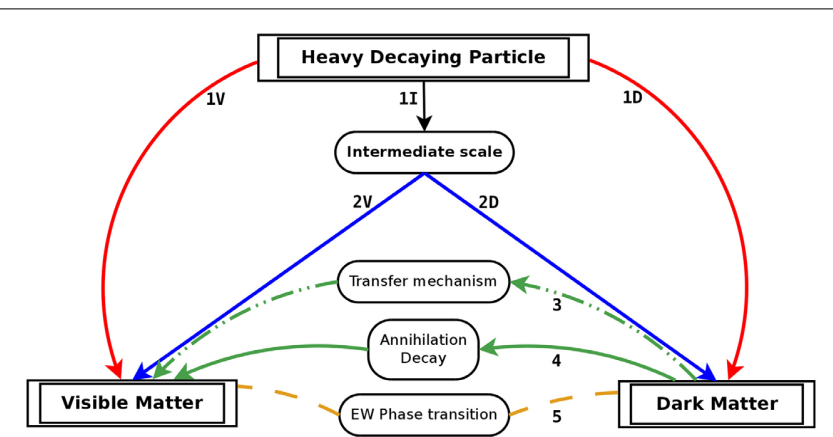

FIGURE 1 | A schematic of the different mechanisms relating DM to baryon asymmetry. The lines are the different stages of the considered mechanism. The labels on the lines are used to describe the model. where $\langle\sigma v\rangle_{\text {f.o. }}$ is the thermal average of the annihilation crosssection of DM times the relative velocity at the time of freeze-out. Which gives the observed abundance for weak interactions crosssections. This coincidence between DM and the weak scale has been dubbed the WIMP miracle. In addition to easily providing the observed relic abundance of DM, the WIMP paradigm is falsifiable. It offers a very rich array of phenomenological tests from underground direct detection experiments to astrophysical signals passing by colliders. Without any doubt, maintaining the success of the WIMP paradigm and extending it to related DM to the baryon asymmetry is an attractive possibility. In this section we review the main theories attaining this goal.

\subsection{ELECTROWEAK BARYOGENESIS}

Electroweak baryogenesis is an appealing minimal scenario of baryogenesis based on the realization of the third Sakharov condition at the electroweak phase transition, see [5] for a review on the mechanism. In the SM a strong first order phase transition, which is necessary in this scenario, requires a very light higgs boson $(<42 \mathrm{GeV})$, moreover the amount of CP violation in the SM is not enough to accommodate the observed BAU. These two considerations imply the need for new physics in order to have a successful baryogenesis and this is where DM comes in. The idea is to use the DM itself (or the dark sector particles) to make this scenario compatible with the SM higgs. A minimal extension of the SM with an extra (complex) scalar [7-11] or two charged singlets [12] achieves this goal, although recent data from LHC and WIMP direct detection experiments render this possibility less attractive because such a DM would have to be sub-dominant (i.e., cannot account for the total density of DM). The same applies for inert

Table 1 | Summary of the models presented in this review and others.

\begin{tabular}{|c|c|c|c|c|c|c|}
\hline Model & DM & HS & BAU & $\mathcal{O}\left(M_{D M}\right)$ & Signal & Diagram \\
\hline EW cogenesis [70] & $\mathcal{W I M P}$ & $x$ & EWPHT & GeV-TeV & $\mathrm{CO}$ & $5^{*}$ \\
\hline WIMPy $Q^{(\dagger)}[22]$ & $\mathcal{W I M P}$ & $x$ & ANNIH & $500 \mathrm{GeV}$ & DD-ID-CO & $T-4^{*}$ \\
\hline Meta-stable WIMP [27] & $\mathcal{W I M P}$ & $x$ & DECAY & GeV-TeV & $\mathrm{CO}$ & $T-4^{*}$ \\
\hline ADM Leptog [59] & $\mathcal{A D \mathcal { M }}$ & $\checkmark$ & DECAY & $\mathrm{KeV}-10 \mathrm{TeV}$ & DD-ID & $1_{V}^{*}{ }^{*} D^{-T}$ \\
\hline Darkogenesis [66] & $\mathcal{A D M}$ & $\checkmark$ & TRANS & $5-15 \mathrm{GeV}$ & GW & $*-3-*$ \\
\hline Baryogenesis from DM [62] & $\mathcal{A D M}$ & $\checkmark$ & TRANS & $3 \mathrm{GeV}$ & DD-CO & $1_{D}^{*}-3-*$ \\
\hline Aidnogenesis [64] & $\mathcal{A D M}$ & $\checkmark$ & DECAY & $6 \mathrm{GeV}$ & DD-FCNC -CO & $1_{V}^{*}-\overline{3}-*-T$ \\
\hline Xogenesis [66] & $\mathcal{A D M}$ & $\checkmark$ & TRANS & $100 \mathrm{GeV}-\mathrm{TeV}$ & $\mathrm{CO}$ & $*-3-*$ \\
\hline
\end{tabular}

The first column shows the type of the DM candidate: WIMP, ADM or NTDM (Non-thermal DM). The second one is about the hidden sector (HS): $\checkmark$ means HS is necessary, $\boldsymbol{x}$ means the model does not rely on HS and $\because$ means that there are realizations of the idea with HS. The third column shows the mechanism that produces the observed baryon asymmetry: EWPHT (Electroweak Phase Transition), DECAY, ANNIH (Annihilation), TRANS (Transfer) or AFDIN (Affleck-Dine). The fourth column gives the order of magnitude of (the sum of) the mass of DM candidate(s). The column Signal shows the predictions of the models: DD (Nuclear recoil direct detection experiments), ID (photons and/or neutrinos indirect detection experiments), CO (Collider), GW (Gravitational waves), IND (Induced proton decay) and FCNC (flavor changing neutral current). Finally the Diagram column shows the diagrammatic route followed by the model following the notation of Figure 1 (see text for details) ( $\left.{ }^{\dagger}\right) L$ refers to the WIMPy baryogenesis scenario applied to leptons, whereas $Q$ refers to the baryonic version. 
higgs extensions of the SM [13] (higher $S U(2)$ representations were considered in $[14,15])$. However, models with vector-like fermions are able to produce the total DM density and BAU for a wide range of masses [16].

An even more extended higgs sector, say a 2-higgs-doublet model improves further the prospects of this scenario by providing the needed CP phases $[17,18]$. There is no direct correlation between DM and baryonic abundances in such theories, however the presence of the dark sector is necessary to have a successful baryogenesis which at the same time constrains the DM mass and couplings. Lastly, LHC and WIMP direct detection experiments may be used to constrain or rule out such a possibility. We note in passing that there are also models based on leptogenesis that follow the same philosophy outlined here, as in [19-21].

\subsection{WIMPY BARYOGENESIS}

Another possibility linking WIMP DM to the baryon asymmetry is the WIMPy baryogenesis model [22]. Here the baryon asymmetry arises from WIMP annihilation instead of the decay of some heavy state like for instance in the usual leptogenesis mechanism. It has been remarked that the annihilation of DM in the early universe can satisfy the Sakharov conditions and leads to a net baryon asymmetry and the observed WIMP relic density.

The baryon asymmetry generated with the WIMP annihilation can be washout from two kind of processes: inverse annihilation of baryons into DM and baryon to antibaryon processes. Therefore, the main requirement for any available WIMPy baryogenesis scenario is that washout processes must freeze-out before that WIMP freeze-out. Inverse annihilations are Boltzmann suppressed for $T<m_{\mathrm{DM}}$ but baryon to antibaryon washout can be relevant also for $T \ll m_{\mathrm{DM}}$. One way to suppress such a processes is by introducing an exotic heavy antibaryon $\psi$ to which WIMP annihilate through the process $D M D M \rightarrow B \psi$ where $B$ is a SM baryon. If the exotic antibaryon $\psi$ has mass $m_{\psi}>m_{\mathrm{DM}}$, for $T<m_{D M}$ its abundance is Boltzmann suppressed and therefore the baryon to antibaryon washout processes are suppressed. So the condition is

$$
m_{\mathrm{DM}} \lesssim m_{\psi} \lesssim 2 m_{\mathrm{DM}}
$$

where the last condition comes from kinematic. B (L) violation is achieved by annihilating the DM to two sectors: baryons (leptons) and exotic antibaryons (antileptons) that are individually asymmetric but together symmetric. It is important that the decay of the exotic particles do not erase the baryon asymmetry generated in the SM sector. For this extra symmetry is required to decouple the exotic fields from the SM.

Solving the model-independent Boltzmann equations for the WIMPy baryogenesis framework, it is possible to show that the baryon asymmetry is proportional to the DM density at the time of freeze-out of the washout processes, i.e., ${ }^{3}$

$$
Y_{\mathrm{B}} \approx \frac{\epsilon}{2}\left[Y_{D M_{\mathrm{wo}}}-Y_{D M}\right]
$$

${ }^{3}$ Here $Y_{X}$ is the ratio of the number density $n_{X}$ of the specie $X$ with the entropy $s$. where $Y_{D M_{\text {wo }}}$ is the DM density at the washout while $Y_{\mathrm{B}, \mathrm{DM}}$ are the observed baryon and DM densities and $\epsilon$ is the baryonantibaryon asymmetry. From Equation (3) and the relation

$$
Y_{D M}=\frac{(5 G e V)}{m_{\mathrm{DM}}} Y_{\mathrm{B}}
$$

it follows that $Y_{D M_{\text {wo }}} \gg Y_{D M}$, namely it is crucial to freeze-out the wash out processes before the WIMP freeze-out temperature otherwise any generated asymmetry would be quickly erased.

As a concrete example we consider a realization of the WIMPy idea in which the WIMP annihilate to leptons generating a lepton asymmetry then converted into a baryon asymmetry through sphaleron like in leptogenesis. The DM candidate consists of a pair of gauge singlet Dirac fermions $Y$ and $\bar{Y}$. In addition to DM two new weak-scale states $\psi$ (fermion $S U_{L}(2)$ doublet) and $S_{1}$ and $S_{2}$ (pseudo-scalar gauge singlets) are added. The fields $\left\{Y, \bar{Y}, \Psi, \bar{\psi}, S_{i}\right\}$ transform under an extra $Z_{4}$ symmetry respectively as $\{+i,-i,-1,-1,-1\}$. The Lagrangian contains the extra terms

$$
\mathcal{L} \supset\left(\lambda_{i} Y^{2}+\lambda_{i}^{\prime} \bar{Y}^{2}\right) S_{i}+\lambda_{\psi_{i}} L \psi S_{i}
$$

Since there is more than one scalar $S_{i}$, it remains a relative complex phase between the $\lambda$ couplings. Then as in the common leptogenesis case the interference between tree level and loop diagrams give rise to CP violation resulting in an asymmetries in $L$ $\left(4^{*}\right)^{4}$ and subsequently converted to B asymmetry by means of the sphalerons. Here differently from leptogenesis, the dark matter $Y$ annihilates into SM leptons $L$ and $\psi$ through the pseudo-scalars $(\mathbf{T})^{5}$ then a lepton asymmetry also accumulate in $\psi$. The processes linking $\psi$ to the SM do not erase the lepton asymmetry thanks to the extra $Z_{4}$ symmetry that decouples $\psi$ from the SM.

At the end an asymmetry is generated from a $2 \rightarrow 2$ process instead of a $1 \rightarrow 2$. An important requirement is that $m_{\Psi}>m_{Y}$ because it implies that the dominant washout process $L \psi \rightarrow$ $L^{\dagger} \psi^{\dagger}$ is Boltzmann-suppressed when DM is annihilating. We summarize diagrammatically the signature of the model as ( $\mathbf{T}-$ $\left.4^{*}\right)$, as it appears in the Table 1 .

The detection prospects are rich in this scenario and include direct (for models with annihilation to quarks), indirect detection (anti-deuteron) and collider signals. See [23, 24] for a general phenomenological study of this class of models. Other models preserving the WIMP miracle and attempting to relate the DM to BAU can be found in $[25,26]$.

\subsection{META-STABLE WIMP}

As in the case of WIMPy baryogenesis, this model [27] attempts to explain the DM/baryon relic density coincidence using the WIMP miracle. The idea is to use a decaying WIMP instead of a stable one. A thermal WIMP $Y$ freeze out at a temperature $T_{f}$ that is typically $T_{f} \sim m_{Y} / 20$. At freeze out the WIMP density is $Y_{Y}\left(T_{f}\right)$ which is equal to the DM density today $Y_{Y}\left(T_{f}\right) \simeq Y_{Y}\left(T_{0}\right)$ if the

\footnotetext{
${ }^{4}$ The depiction of this step in the schematic: the DM annihilates into the visible sector (line 4 ), with the $*$ is there to show that an asymmetry is produced in this step.

${ }^{5}$ We use here the letter T to emphasize that the DM is thermally produced.
} 
WIMP is stable. The authors consider two kinds of WIMPs: one stable $Y_{1}$ that is the DM candidate and one $Y_{2}$ that decay after freeze out, with the densities of the two WIMPS at the freeze out being almost the same $Y_{Y_{1}}\left(T_{f}\right) \approx Y_{Y_{2}}\left(T_{f}\right)$. The density of the decay WIMP at the freeze out temperature is the initial condition for the baryogenesis.

The meta-stable WIMP $Y_{2}$ decays after thermal freeze-out into baryons in such a way that the baryon number B and CP are violated. In a minimal realization of the idea, the SM is extended to include a di-quark scalar $\phi$ and $\psi$ which are Majorana fermions and a singlet scalar $S$. The relevant couplings are

$$
\phi d d, \quad Y_{2} \bar{u} \phi, \quad \psi \bar{u} \phi, \quad Y_{2}^{2} S, \quad|H|^{2} S
$$

Where $u$ and $d$ are the SM quarks. The scalar $S$ mediates the thermal annihilation of $Y_{2} Y_{2}$ into SM. The meta-stable WIMP decay as $Y_{2} \rightarrow u \phi^{*}$ followed by the decay of $\phi \rightarrow d d$. A CP asymmetry $\epsilon_{C P}$ in $Y_{2} \rightarrow u \phi^{*}$ and $\bar{Y}_{2} \rightarrow \bar{u} \phi$ arises from the interference between the tree-level diagram with the one loop diagram mediated by $\psi$ (that shares with $Y_{2}$ the same quantum numbers). In order to generate a baryon asymmetry the WIMP must decay before the BBN and after WIMP freeze out, i.e., $T_{B B N}<T_{Y_{2}}<$ $T_{f}$. Solving the Boltzmann equations it is possible to find the baryon density today

$$
Y_{B}\left(T_{0}\right) \approx \epsilon_{C P} \int_{T_{0}}^{T_{D}} \frac{d Y_{Y_{2}}}{d T} d T \simeq \epsilon_{C P} Y_{Y_{2}}\left(T_{f}\right)
$$

Using the relations $Y_{Y}\left(T_{f}\right) \approx Y_{Y_{2}}\left(T_{f}\right)$ and that $Y_{Y}\left(T_{f}\right) \simeq Y_{Y}\left(T_{0}\right)$ we arrive at the result

$$
\Omega_{B}=\epsilon_{C P} \frac{m_{p}}{m_{D M}} \Omega_{D M}
$$

where $\Omega_{D M}$ is the relic abundance of the DM. The model lies at the electroweak scale and therefore it can be probed in colliders.

\section{ASYMMETRIC DARK MATTER MODELS}

ADM [28-35] is a class of DM models often seen as an alternative to the WIMP paradigm. The rationale of ADM is based on the hypothesis that DM abundance is, similarly to baryons, only the surviving asymmetric part of the initial density and is of the same order as the baryon asymmetry, i.e.,

$$
n_{Y}-n_{\bar{Y}} \sim n_{b}-n_{\bar{b}}
$$

where $Y$ denotes the DM particle. The motivation comes from the fact that the observed DM and visible matter abundances are remarkably close to each other. These models usually lead to a relation between DM mass and proton mass: $M_{D M} \sim 5 M_{P}$ in contrast with WIMP DM models where the scale of reference is the weak scale. The relation between the DM mass and the proton mass is however not explained except in some models based on hidden sectors such as in mirror worlds [36-38], models with a dark QCD [39] or composite models (see below).

$\mathrm{ADM}$ can be implemented in many ways leading to a very rich theoretical and phenomenological landscape. While it is difficult to classify these models in a straightforward way, it is nevertheless enriching to highlight the key principles they usually rely on. Basically two main approaches are followed: (1) Dark and visible matter asymmetries are generated at the same time. This is usually achieved with the decay of a heavy particle. (2) The asymmetry is generated in the dark sector then is transferred (via sphaleron processes, higher dimension operators or renormalizable interactions) to the visible sector or vice versa. It is also necessary to pass at some point by a thermalization phase to get rid or to avoid the production of the symmetric part of DM (a less extreme cancelation of the asymmetric part leads to mixed scenarios between WIMP and ADM [40]).

We will present here ADM models explicitly showing the key assumptions and principles used as well as their phenomenological impact. They make use of the main ADM concepts and pass by the main diagrammatic roads. For a recent review and an exhaustive list of reference we refer the reader to [41-43] and for a more succinct overview [44].

\subsection{COMPOSITE ADM}

The idea of the ADM has been proposed in the seminal work of Nussinov [28] who suggested that in analogy with the visible sector's baryon asymmetry, a technibaryon asymmetry is a natural possibility. This idea has been recently revamped in the context of walking dynamics [45-48]. If the model is arranged such that the lightest technibaryon (LTB) is neutral and stable, the density of the LTB scales as:

$$
\frac{\Omega_{T B}}{\Omega_{B}}=\frac{T B}{B} \frac{m_{T B}}{m_{p}}
$$

Where $m_{p}$ is the proton mass, $m_{T B}$ is the mass of the LTB. TB and $B$ are the technibaryon and baryon number densities, respectively. This is the typical scaling of ADM models.

The model discussed in [45] is a technicolor theory based on the $S U(4)$ global symmetry spontaneously broken down to $S O(4)$. Such a breaking gives rise to 9 Goldston bosons, three of them corresponding to the SM gauge bosons. The remmant six Goldstone bosons carry technibaryon charge and the lightest of them (LTB) is the DM candidate ${ }^{6}$. In $[49,50]$ the properties of composite (asymmetric or symmetric) dark matter candidates have been computed in detail via first-principle lattice simulations.

\subsection{KITANO-LOW}

The model implemented in [34] considers a mechanism originally proposed in [31] to unify in an elegant way the abundances of DM and baryons. It is a prototype of the ADM models based on decay of a field connecting the dark and visible sectors.

The authors postulate a new symmetry, namely a $Z_{2}$ parity, under which the SM particles are neutral and new particles are charged, forming a dark or hidden sector. The lightest of the hidden particle is stable and is a DM candidate. A generalized B-L number is unbroken and is shared between the SM and the dark sector, thus any excess of B-L that is generated in one of the two

\footnotetext{
${ }^{6}$ The Goldstone bosons are supposed to pick up a mass from a higher scale.
} 
sectors is compensated by the same excess in the other sector. After baryogenesis the interactions between the visible and the dark sectors become negligible and the B-L excesses are separately conserved in the two sectors giving a relation between the visible and dark relic densities.

A simple model realizing the idea consists of a heavy particle $P$, a messenger particle $X$ which carries a color charge and the DM candidate $Y$, all odd under the $Z_{2}$ while the SM is even. The mechanism passes through 3 stages. In the first stage $P$ has CP-violating out of equilibrium decays into SM and to a lighter messenger $X$ generating an excess in both sectors but preserving the generalized $\mathrm{B}-\mathrm{L}$ globally. Then is assumed that below the baryogenesis temperature the two sector are decoupled and the two asymmetries are conserved such that we have:

$$
n_{\mathrm{B}-\mathrm{L}}^{\mathrm{SM}}=-n_{\mathrm{B}-\mathrm{L}}^{X} \sim n_{X}-n_{\bar{X}}
$$

In the second stage the dark $X$ messenger annihilate away its symmetric part with $\bar{X}$ through gauge interactions and we are left with its asymmetric part only. In the third and final stage the decay of $X$ to DM particle $Y$ and therefore

$$
n_{\mathrm{DM}} \propto n_{\mathrm{B}-\mathrm{L}}^{X}
$$

giving a tight relation between the visible (baryonic) and DM number densities. To ensure that such a relation exists it is important that $X$ is long lived enough such that it decays after its symmetric part cancels out.

We summarize the mechanism: Decay of $P$ that produces the asymmetries in $X\left(\mathbf{1}_{I}^{*}\right)$ and $\mathrm{SM}\left(\mathbf{1}_{V}^{*}\right.$, since an asymmetry in the visible sector is generated by the decay) followed by symmetric annihilation of $X(\mathbf{T})$ and finally the decay of $X$ to the lightest dark particle $Y\left(\mathbf{2}_{D}\right)$. We denote the full mechanism in a compact way as $\left(\mathbf{1}_{V}^{*}-\mathbf{1}_{I}^{*}-\mathbf{T}-\mathbf{2}_{D}\right)$.

An interesting possibility is to consider $X$ itself as the DM particle. This possibility is not possible here here because of charge assignment of $X$ (colored particle). However, we will see now that Hylogenesis realizes this possibility. Note that the original asymmetry can be generated through the Affleck-Dine [51,52] mechanism in a SUSY framework [53-56] or through leptogenesis as in [57].

\subsection{HYLOGENESIS}

This model [58] is based on a hidden sector composed of 3 Dirac fermions $X_{1}, X_{2}, Y$ and a complex scalar $\phi$. It is assumed that $M_{\phi} \sim M_{Y} \sim G e V$ and $T e V<M_{X_{1}}<M_{X_{2}}$. $X_{i}$ are made to couple to the visible sector through the neutron portal $\left(X_{i} d^{c} u^{c} d^{c}\right)$, the relevant terms in the Lagrangian are:

$$
\mathcal{L} \supset \frac{\lambda_{i}}{\Lambda^{2}} X_{i} d^{c} u^{c} d^{c}+\kappa_{i} \bar{X}_{i} Y \Phi+\text { h.c. }
$$

The particle content and the symmetries of the model permit the definition of a generalized baryon number (B), conserved by both sectors, under which $B_{X}=-\left(B_{Y}+B_{\Phi}\right)=1$ as well as non-reducible CP phases.

In the early universe an equal number of $X_{1}$ and its antiparticle $\bar{X}_{1}$ are generated non-thermally (e.g. during reheating) and the total baryon number is zero at this stage. Then both states $X_{1}$ and $\bar{X}_{1}$ decay into the visible and hidden states as $X_{1} \rightarrow u d d\left(\mathbf{1}_{V}^{*}\right)$ and $X_{1} \rightarrow \bar{Y} \Phi^{*}\left(\mathbf{1}_{D}^{*}\right)$ and their conjugates at tree level and through loops (including the lighter dark particles $\phi$ and $Y$ ), generating an asymmetry in the visible sector $\epsilon_{V}$ and an asymmetry in the hidden sector $\epsilon_{D}$ á la leptogenesis

$$
\epsilon_{V}=\frac{\Gamma\left(X_{1} \rightarrow u d d\right)-\Gamma\left(\bar{X}_{1} \rightarrow \bar{u} \bar{d} \bar{d}\right)}{\Gamma_{X_{1}}} \simeq \frac{m_{X_{1}}^{5} \operatorname{Im}\left(\lambda_{1}^{*} \lambda_{2} \kappa_{1} \kappa_{2}^{*}\right)}{256 \pi^{3}\left|\kappa_{1}\right|^{2} \Lambda^{4} m_{X_{2}}}
$$

where $\Gamma_{X_{1}}$ is the total rate and $\epsilon_{D}$ can be obtained in a similar way. We have

$$
\begin{aligned}
& \Gamma\left(X_{1} \rightarrow \text { udd }\right)=\Gamma_{V}+\epsilon_{V} \Gamma_{X_{1}}, \\
& \Gamma\left(\bar{X}_{1} \rightarrow \bar{u} \bar{d} \bar{d}\right)=\Gamma_{V}-\epsilon_{V} \Gamma_{X_{1}}, \\
& \Gamma\left(X_{1} \rightarrow \bar{Y} \Phi^{*}\right)=\Gamma_{D}-\epsilon_{D} \Gamma_{X_{1}}, \\
& \Gamma\left(\bar{X}_{1} \rightarrow Y \Phi\right)=\Gamma_{D}+\epsilon_{D} \Gamma_{X_{1}} .
\end{aligned}
$$

Because $X_{1}$ is a Dirac particle, the asymmetry generated in the visible sector is then translated as an asymmetry in the hidden sector. Indeed, CPT invariance forces the particle and its anti-particle to have equal total decay rates $\Gamma\left[X_{1} \rightarrow n+\bar{Y} \Phi^{*}\right]=\Gamma\left[\bar{X}_{1} \rightarrow\right.$ $\bar{n}+Y \Phi]$, which translates as a relation between asymmetries, that is $\epsilon_{D}=-\epsilon_{V}$ where we have used the Equation (15). Therefore, in the decay of $X_{1}$ and $\bar{X}_{1}$ a baryon number is generated in the visible sector and an equal and opposite baryon number is generated in the hidden sector so that the total baryon number is zero. The two asymmetries are frozen-in thanks to the weakness of the interactions between the two sectors.

The final step is to cancel out the symmetric part of the dark matter particles and this is achieved for instance with an extra $U(1)_{D}$ gauge symmetry in the hidden sector under which $Y$ and $\Phi$ have opposite charges and $X_{1,2}$ are neutral. The symmetric part is depleted $(\mathbf{T})$ by the annihilation processes $Y \bar{Y} \rightarrow Z^{\prime} Z^{\prime}$ and $\Phi \Phi^{*} \rightarrow Z^{\prime} Z^{\prime}$ with $m_{Z^{\prime}}<m_{Y, \Phi} \sim G e V$ (this is consistent with present observations for $10^{-6}<\kappa<10^{-2}$ ) with $Z^{\prime}$ decaying to $\mathrm{SM}$ through photon. These cross section are much larger to the one need to obtain the correct DM relic density by thermal freezeout. Then the DM density is given by the residual asymmetric component and we are then left with the relation:

$$
n_{Y}=n_{\Phi}=n_{B}
$$

that gives a strong relation between the visible and dark matter abundances:

$$
\frac{\Omega_{D M}}{\Omega_{B}}=\frac{\left(M_{Y}+M_{\Phi}\right)}{M_{P}} \sim 5 .
$$

We denote in a compact way this mechanism with the signature $\left(\mathbf{1}_{V}^{*}-\mathbf{1}_{D}^{*}-\mathbf{T}\right)$. Because of the neutron portal, hylogenesis provides an interesting signature of the DM: the induced proton decay (IND). Indeed DM can scatter with protons producing mesons $\phi^{*} p \rightarrow Y K^{+}$.

\subsection{ADM FROM LEPTOGENESIS}

If we take Majorana instead of Dirac decaying fields in the previous model, we get different consequences on the DM mass. The model considered in [59] is based on the decay of a heavy right handed neutrino field $N$. 
The model is an extension of the SM and consists of two righthanded neutrinos and a scalar $\phi$ and fermion $Y$ gauge singlets, charged under an extra $Z_{2}$ parity, that made the hidden sector

$$
\mathcal{L} \supset M_{i} N_{i}^{2}+y_{i} L H N_{i}+\lambda_{i} N_{i} Y \phi+\text { h.c. }
$$

$N$ couples to the SM with Dirac Yukawa coupling and to the hidden sector, $Y$ is the DM candidate. Therefore, $N$ can decay (out of equilibrium) simultaneously as $N \rightarrow L H\left(\mathbf{1}_{V}^{*}\right)$ and $N \rightarrow Y \phi$ $\left(\mathbf{1}_{D}^{*}\right)$ generating two different and unrelated CP-asymmetries $\epsilon_{L}$ and $\epsilon_{D M}$ respectively. Here $N$ is a Majorana particle and CPT does not imply that $\left|\epsilon_{L}\right|=\left|\epsilon_{D M}\right|$ like in Hylogenesis (see previous section). The DM must be a Dirac particle in order to preserve a lepton number. Because both $Y$ and $\phi$ are charged under the extra $Z_{2}$, the DM is stable and the hidden sector can interact with the SM only by means of the heavy right-handed neutrino. In order to cancel out the symmetric component of the DM, an additional gauged $U(1)$ interaction is imposed to annihilate the $Y, \bar{Y}$ pair. We are left with the asymmetric parts of $Y(\mathbf{T})$. The DM and baryon density $\Omega_{D M} / \Omega_{B}$ is then proportional to the ratio of the CP-asymmetries $\epsilon_{D M} / \epsilon_{L}$

$$
\frac{\Omega_{\mathrm{DM}}}{\Omega_{B}} \sim \frac{m_{\mathrm{DM}}}{m_{p}} \frac{\epsilon_{D M}}{\epsilon_{L}} \frac{\eta_{D M}}{\eta_{L}}
$$

where $\eta_{D M, L}$ are the washout factor. Therefore, the DM mass can be very different from the value of $5 M_{p}$ given in most ADM models. A similar model based on type-II leptogenesis instead of type-I has been proposed in [60]. See also [57] for an earlier ADM model based on leptogenesis and where the DM mass is in the typical few $\mathrm{GeV}$ scale.

\subsection{DARKOGENESIS}

In this model [61] an asymmetry is generated in the dark sector and is then transferred to the visible sector. The DM asymmetry arises from a first order dark phase transition in the hidden sector to which the SM does not participate. The dark baryogenesis proceed through the symmetry breaking phase transition of a dark non-Abelian gauge group $G_{D}$. The fields in the dark sector have a global dark symmetry $U_{D}(1)$ which is anomalous under $G_{D}$. During the symmetry breaking first order phase transition an dark asymmetry is generated by means of CP violating interactions.

The asymmetry can be transferred to the visible sectors in two ways: by fields that carry both hidden and visible charges (perturbatively) or via electroweak sphalerons (non-perturbatively).In the last case, in order to transmit the asymmetry from the dark sector to the SM one, it is required a mediator charged under both the $S U_{L}(2)$ and the dark symmetry $U_{D}(1)$. Then the dark number is anomalous under $S U_{L}(2)$ and the $S M$ electroweak sphaleron can convert the asymmetry of the dark sector into an asymmetry in the SM.

In the first case the connectors can consists of higher order effective operators of the type

$$
O_{d} L H, \quad O_{d} u d d, \quad O_{d} L L e, \quad O_{d} L Q d, \quad O_{d} L H L H,
$$

where $O_{d}$ is a dark sector operator like for instance $O_{d}=X, X^{2}$. The hidden sector phase transition occurs at a temperature above the temperature at which the effective transfer operator freezeout The dark matter mass lies around 5 to 15 times the mass of the proton.

Direct detection cannot falsify the darkogenesis mechanism, however the gravitational wave signal from dark first order transition could in principle probe this mechanism. The asymmetry in the dark sector can also be generated via a different baryogenesis mechanism, see [62] for an example where a heavy particle decays to the dark sector, creating an asymmetry there that is then transferred to the visible sector. For the opposite case, see [63] or aidnogenesis[64], for instance where the asymmetry is transferred through sphalerons from the SM to the dark sector. Diagrammatically we denote this model as : ${ }^{*}-3-^{*}$, which means that an original asymmetry in the dark sector (following the direction the arrow) is transferred to the visible sector. See also [65] for a recent model where sphalerons are responsible for cogenerating the dark matter.

\subsection{XOGENESIS}

Like in the darkogenesis model, here [66] a DM asymmetry is created and then transferred to the baryon by means of transfer operators. The problem of the creation of a DM asymmetry is not addressed here and the authors focuses on the transfer mechanisms. The main difference between this mechanism and the classic ADM ones going in the same direction is that the DM mass can be around the weak scale instead of the proton mass (for a different idea how to obtain heavy ADM see [67]) without fine-tuning the parameters. The main idea can be summarized as follows: If DM is not relativistic at the temperature where the transfer operator decouples $T_{D}$ then the DM number density undergoes a thermal suppression allowing the DM to be heavy.

The transfer can be due from the $S U_{L}(2)$ sphalerons (or the exotic sphalerons of a new gauge group) or lepton/baryon number violation from higher order operators. In any transfer scenario chemical equilibrium between DM and baryon is maintained until the transfer operator decouples. When the transfer is active, we have:

$$
\mu_{D M} \sim \mu_{B}
$$

Given a specie $i$ in general its asymmetry $n_{\Delta i}=n_{i}-\bar{n}_{i}$ is proportional to its chemical potential

$$
n_{i}=c_{i} \mu_{i}
$$

The coefficients $c_{i}$ are function of the mass and temperature $c_{i}=$ $c_{i}\left(m_{i}, T\right)[29]:$

$$
c_{i}=g_{i} f\left(m_{i} / T\right) T^{2} R^{3}, f(x)=\frac{1}{4 \pi^{2}} \int_{0}^{\infty} \frac{y^{2} d y}{\cosh ^{2}\left(\sqrt{x^{2}+y^{2}} / 2\right)}
$$

where $g_{i}$ is the statistical weight and $R$ is the Robertson-Walker scale factor at temperature $T$. For small value of $m_{i} / T$ then 
$f\left(m_{i} / T\right)$ tend to a constant, while for large $m_{i} / T$ then $f\left(m_{i} / T\right)$ is very small

$$
f\left(m_{i} / T\right) \sim\left[\begin{array}{ll}
m_{i} / T \ll 1, & 1 / 6 \\
m_{i} / T \gg 1, & 2(m / 2 \pi T)^{3 / 2} e^{-m / T}
\end{array}\right]
$$

Typically only the first possibility where $m_{D M} / T_{D} \ll 1$ is taken ( $T_{D}$ is the decoupling temperature of the transfer operator). In this case from Equations (21) and (22) it follows that $n_{\Delta_{D M}} \sim n_{\Delta_{B}}$ leading to the 'prediction' $m_{D M} \sim 5 m_{p}$. However, a second solution is possible. If the ratio $m_{D M} / T_{D}$ is large, then the coefficients $c_{D M}$ is suppressed, see Equations (22) and (24). This results in a lower $n_{D M}$ with respect to the case where the ratio $m_{i} / T_{D}$ is small and thus a larger DM is allowed. For a given value of $T_{D}$ the non-relativistic solution give about $m_{D M} \sim 10 T_{D}$ instead of $5 \mathrm{GeV}$ (relativistic solution), giving a mass for the DM of the order of the $\mathrm{TeV}$.

A simple example is given by a DM particle $Y$ that transform as a fermion doublet of $S U_{L}(2)$ with hypercharge $+1 / 2$. Since the $\mathrm{DM}$ is charged under $S U_{L}(2)$, it interacts with the $\mathrm{SM}$ sphaleron. Thanks to the sphaleron $Y$ and quarks are in thermal equilibrium, therefore the DM and quarks chemical potential are releted, i.e., $\mu_{Y}=-3 \mu_{u_{L}}$. In this example the decoupling temperature $T_{D}$ of the transfer operator is the temperature where the spahleron is no more active, that is around $200 \mathrm{GeV}$. Solving equations (21) and (22) one gets for the DM a value of about $2000 \mathrm{GeV}$.

The idea has been illustrated with different classes of transfer operators: SM sphalerons, exotic sphalerons of a new gauge group and lepton or baryon number violation higher order operators. Since the DM is heavy it will be difficult to search for it but new particles at the weak or $\mathrm{TeV}$ scale are can be probed in collider experiments.

\section{NON-THERMAL DARK MATTER MODELS 4.1. CLADOGENESIS}

Cladogenesis [68] is based on the observation that the dilution factor due to entropy release by moduli decay is very close to the observed baryon asymmetry. Indeed for a modulus $\tau$ with a reheating temperature in the range $\mathrm{MeV}-\mathrm{GeV}$ (corresponding to $M_{\tau}$ of order $20-1000 \mathrm{TeV}$ ) the dilution factor is given by

$$
Y_{\tau}=\frac{3 T_{R H}}{4 M_{\tau}^{4}} \sim 10^{-9}-10^{-7}
$$

a value that is close to $\eta_{B}$ and also to $Y_{D M}$ as long as $M_{D M}$ is within a factor or two from the proton mass. At the same time any previous DM abundance will be suppressed by the same factor. These considerations lead the authors of Cladogenesis to consider a non-thermal origin of DM from modulus decay. The scenario goes as follow: $\tau$ decays to some species $N\left(\mathbf{1}_{I}\right)$ and to DM (directly or via dark sector particles following $\mathbf{1}_{D}$ ). The decay to DM must be suppressed down to $10^{-3}$ to achieve the observed relic abundance. $N$ then decays to SM by violating baryon (or lepton) number and $\mathrm{CP}$ to produce the correct baryon asymmetry $\left(2_{V}^{*}\right)$. Note that the DM is not asymmetric in this model because baryogenesis is done in the visible sector only.
Another example of non-thermal mechanism is given in [69] where the DM arises from the out-off equilibrium decay of the inflaton instead of the moduli.

\section{SUMMARY}

In this short review we have given an overview of the models linking the generation of the baryon asymmetry of the universe and dark matter. These models are varied and diverse and tackle the problematic from different points of view. Models attempting to preserve the WIMP miracle lead to a very rich phenomenology and their couplings can be probed at LHC soon. These models do not address the coincidence between the baryon and DM asymmetries and the link between the two abundances is not strong. ADM models, one the other hand, give a natural explanation to this ratio at the price of WIMP phenomenology. Lastly nonthermal production models are yet another possibility relating the genesis of the dark and visible sector. LHC and dark matter search experiments will probe large chunks from the theoretical landscape of DM, hopefully shedding light on its nature and on the mechanism at work for baryogenesis.

We summarize the models discussed here in Table 1 where we give information about the nature of their DM, the BAU mechanism at work, the existence of a hidden sector, range of the DM mass allowed in the model as well as the expected signal. The last column shows the diagrammatic signature of the model based on Figure 1 and the convention outlined in the introduction.

\section{ACKNOWLEDGMENTS}

Stefano Morisi thanks to DFG grant WI 2639/4-1 for financial support. Sofiane. M. Boucenna was supported by the Spanish MINECO under grants FPA2011-22975 and MULTIDARK CSD2009-00064 (Consolider-Ingenio 2010 Programme), by Prometeo/2009/091 (Generalitat Valenciana), by the EU ITNUNILHC PITN-GA-2009-237920.

\section{REFERENCES}

1. Ade PAR, Aghanim N, Armitage-Caplan C, Arnaud M, Ashdown M, AtrioBarandela F. et al. Planck 2013 Results. XVI. Cosmological Parameters. (2013) arXiv: 1303.5076

2. Bertone G, Hooper D, Silk J. Particle dark matter: evidence, candidates and constraints. Phys Rept. (2005) 405:279-390. doi: 10.1016/j.physrep.2004.08.031

3. Bergstrom L. Nonbaryonic dark matter: observational evidence and detection methods. Rept Prog Phys. (2000) 63:793. doi: 10.1088/0034-4885/63/5/2r3

4. Dine M, Kusenko A. The origin of the matter - antimatter asymmetry. Rev Mod Phys. (2003) 76:1. doi: 10.1103/RevModPhys.76.1

5. Riotto A, Trodden M. Recent progress in baryogenesis. Ann Rev Nucl Part Sci. (1999) 49:35-75. doi: 10.1146/annurev.nucl.49.1.35

6. Sakharov AD. Violation of CP invariance, $c$ asymmetry, and baryon asymmetry of the universe. Pisma ZhEksp Teor Fiz. (1967) 5:32-5.

7. Profumo S, Ramsey-Musolf MJ, Shaughnessy G. Singlet higgs phenomenology and the electroweak phase transition. JHEP (2007) 0708:010. doi: 10.1088/1126-6708/2007/08/010

8. Espinosa JR, Konstandin T, Riva F. Strong electroweak phase transitions in the standard model with a singlet. Nucl Phys. (2012) B854:592-630. doi: 10.1016/j.nuclphysb.2011.09.010

9. Barger V, Langacker P, McCaskey M, Ramsey-Musolf M, Shaughnessy G. Complex singlet extension of the standard model. Phys Rev. (2009) D79:015018. doi: 10.1103/PhysRevD.79.015018

10. Cline JM, Kainulainen K. Electroweak baryogenesis and dark matter from a singlet higgs. JCAP (2013) 1301:012. doi: 10.1088/1475-7516/2013/01/012

11. Ahriche A, Nasri S. Light dark matter, light higgs and the electroweak phase transition. Phys Rev. (2012) D85:093007. doi: 10.1103/PhysRevD.85.093007 
12. Ahriche A, Nasri S. Dark matter and strong electroweak phase transition in a radiative neutrino mass model. JCAP (2013) 1307:035. doi: 10.1088/14757516/2013/07/035

13. Cline JM, Kainulainen K. Improved electroweak phase transition with subdominant inert doublet dark matter. Phys Rev. (2013) D87:071701. doi: 10.1103/PhysRevD.87.071701

14. Chowdhury TA, Nemevsek M, Senjanovic G, Zhang Y. Dark matter as the trigger of strong electroweak phase transition. JCAP (2012) 1202:029. doi: 10.1088/1475-7516/2012/02/029

15. AbdusSalam SS, Chowdhury TA. Scalar representations in the light of electroweak phase transition and cold dark matter phenomenology. (2013) arXiv: 1310.8152 .

16. Fairbairn M, Grothaus P. Baryogenesis and dark matter with vector-like fermions. JHEP (2013) 1310:176. doi: 10.1007/JHEP10(2013)176

17. McLerran LD, Shaposhnikov ME, Turok N, Voloshin MB. Why the baryon asymmetry of the universe is approximately $10^{* *}-10$. Phys Lett. (1991) B256:451-6.

18. Cline JM, Kainulainen K, Trott M. Electroweak baryogenesis in two higgs doublet models and B meson anomalies. JHEP (2011) 1111:089. doi: 10.1007/JHEP11(2011)089

19. Basso L, Fischer O, van der Bij JJ. A natural $Z^{\prime}$ model with inverse seesaw and leptonic dark matter. Phys Rev. (2013) D87:035015. doi: 10.1103/PhysRevD.87.035015

20. Canetti L, Drewes M, Shaposhnikov M. Sterile neutrinos as the origin of dark and baryonic matter. Phys Rev Lett. (2013) 110:061801. doi: 10.1103/PhysRevLett.110.061801

21. Canetti L, Drewes M, Frossard T, Shaposhnikov M. Dark matter, baryogenesis and neutrino oscillations from right handed neutrinos. Phys Rev. (2013) D87:093006. doi: 10.1103/PhysRevD.87.093006

22. Cui Y, Randall L, Shuve B. A WIMPy baryogenesis miracle. JHEP (2012) 1204:075. doi: 10.1007/JHEP04(2012)075

23. Bernal N, Josse-Michaux FX, Ubaldi L. Phenomenology of WIMPy baryogenesis models. JCAP (2013) 1301:034. doi: 10.1088/1475-7516/2013/01/034

24. Bernal N, Colucci S, Josse-Michaux FX, Racker J, Ubaldi L. On baryogenesis from dark matter annihilation. (2013). JCAP (2013) 1310:035. doi: 10.1088/1475-7516/2013/10/035

25. Davidson S, Elmer M. Similar dark matter and baryon abundances with $\mathrm{TeV}$ scale leptogenesis. JHEP (2012) 1210:148. doi: 10.1007/JHEP10(2012)148

26. McDonald J. Baryomorphosis: relating the baryon asymmetry to the "WIMP miracle.” Phys Rev. (2011) D83:083509. doi: 10.1103/PhysRevD.83.083509

27. Cui Y, Sundrum R. Baryogenesis for weakly interacting massive particles. Phys Rev. (2013) D87:116013. doi: 10.1103/PhysRevD.87.116013

28. Nussinov S. Technocosmology: could a technibaryon excess provide a "natural" missing mass candidate? Phys Lett. (1985) B165:55.

29. Barr SM, Chivukula RS, Farhi E. Electroweak fermion number violation and the production of stable particles in the early universe. Phys Lett. (1990) B241:387-91.

30. Barr SM. Baryogenesis, sphalerons and the cogeneration of dark matter. Phys Rev. (1991) D44:3062-6.

31. Kuzmin VA. A Simultaneous solution to baryogenesis and dark matter problems. Phys Part Nucl. (1998) 29:257-65. doi: 10.1134/1.953070

32. Hooper D, March-Russell J, West SM. Asymmetric sneutrino dark matter and the Omega(b) / Omega(DM) puzzle. Phys Lett. (2005) B605:228-36. doi: 10.1016/j.physletb.2004.11.047

33. Farrar GR, Zaharijas G. Dark matter and the baryon asymmetry of the universe. (2004) Phys Rev. Lett. (2006) 96:041302. doi: 10.1103/PhysRevLett.96.041302

34. Kitano R, Low I. Dark matter from baryon asymmetry. Phys Rev. (2005) D71:023510. doi: 10.1103/PhysRevD.71.023510

35. Kaplan DE, Luty MA, Zurek KM. Asymmetric dark matter. Phys Rev. (2009) D79:115016. doi: 10.1103/PhysRevD.79.115016

36. Foot R, Volkas RR. Explaining omega(Baryon) approximately 0.2 omega(Dark) through the synthesis of ordinary matter from mirror matter: a more general analysis. Phys Rev. (2004) D69:123510. doi: 10.1103/PhysRevD.69.123510

37. Hodges HM. Mirror baryons as the dark matter. Phys Rev. (1993) D47:456-9.

38. Mohapatra RN, Teplitz VL. Mirror dark matter. Phys Rev. (2000) D62:063506.

39. Bai Y, Schwaller P. The Scale of Dark QCD. (2013) arXiv: 1306.4676.

40. Graesser ML, Shoemaker IM, Vecchi L. Asymmetric WIMP dark matter. JHEP (2011) 1110:110. doi: 10.1007/JHEP10(2011)110
41. Sannino F. Conformal dynamics for TeV physics and cosmology. Acta Phys Polon. (2009) B40:3533-743.

42. Petraki K, Volkas RR. Review of asymmetric dark matter. Int J Mod Phys. (2013) A28:1330028. doi: 10.1142/S0217751X13300287

43. Zurek KM. Asymmetric dark matter: theories, signatures, and constraints. (2013) arXiv: 1308.0338

44. Davoudiasl H, Mohapatra RN. On relating the genesis of cosmic baryons and dark matter. New JPhys. (2012) 14:095011. doi: 10.1088/1367-2630/14/9/095011

45. Gudnason SB, Kouvaris C, Sannino F. Towards working technicolor: effective theories and dark matter. Phys Rev. (2006) D73:115003. doi: 10.1103/PhysRevD.73.115003

46. Gudnason SB, Kouvaris C, Sannino F. Dark matter from new technicolor theories. Phys Rev. (2006) D74:095008. doi: 10.1103/PhysRevD.74.095008

47. Nardi E, Sannino F, Strumia A. Decaying dark matter can explain the e+excesses. JCAP (2009) 0901:043. doi: 10.1088/1475-7516/2009/01/043

48. Del Nobile E, Kouvaris C, Sannino F. Interfering composite asymmetric dark matter for DAMA and CoGeNT. Phys Rev. (2011) D84:027301. doi: 10.1103/PhysRevD.84.027301

49. Lewis R, Pica C, Sannino F. Light asymmetric dark matter on the lattice: $\mathrm{SU}(2)$ technicolor with two fundamental flavors. Phys Rev. (2012) D85:014504. doi: 10.1103/PhysRevD.85.014504

50. Hietanen A, Lewis R, Pica C, Sannino F. Composite goldstone dark matter: experimental predictions from the lattice. (2013) arXiv: 1308.4130.

51. Affleck I, Dine M. A new mechanism for baryogenesis. Nucl Phys. (1985) B249:361.

52. Allahverdi R, Mazumdar A. A mini review on affleck-dine baryogenesis. New J Phys. (2012) 14:125013. doi: 10.1088/1367-2630/14/12/125013

53. Bell NF, Petraki K, Shoemaker IM, Volkas RR. Pangenesis in a baryonsymmetric universe: dark and visible matter via the affleck-dine mechanism. Phys Rev. (2011) D84:123505. doi: 10.1103/PhysRevD.84.123505

54. Cheung C, Zurek KM. Affleck-dine cogenesis. Phys Rev. (2011) D84:035007. doi: 10.1103/PhysRevD.84.035007

55. von Harling B, Petraki K, Volkas RR. Affleck-dine dynamics and the dark sector of pangenesis. JCAP (2012) 1205:021. doi: 10.1088/1475-7516/2012/05/021

56. Roszkowski L, Seto O. Axino dark matter from Q-balls in affleck-dine baryogenesis and the omega(b) - omega(DM) coincidence problem. Phys Rev Lett. (2007) 98:161304. doi: 10.1103/PhysRevLett.98.161304

57. Cosme N, Lopez Honorez L, Tytgat MHG. Leptogenesis and dark matter related? Phys Rev. (2005) D72:043505. doi: 10.1103/PhysRevD.72.043505

58. Davoudiasl H, Morrissey DE, Sigurdson K, Tulin S. Hylogenesis: a Unified Origin for Baryonic Visible Matter and Antibaryonic Dark Matter. Phys Rev Lett. (2010) 105:211304. doi: 10.1103/PhysRevLett.105.211304

59. Falkowski A, Ruderman JT, Volansky T. Asymmetric dark matter from leptogenesis. JHEP (2011) 1105:106. doi: 10.1007/JHEP05(2011)106

60. Arina C, Sahu N. Asymmetric inelastic inert doublet dark matter from triplet scalar leptogenesis. Nucl Phys. (2012) B854:666-99. doi: 10.1016/j.nuclphysb.2011.09.014

61. Shelton J, Zurek KM. Darkogenesis: a baryon asymmetry from the dark matter sector. Phys Rev. (2010) D82:123512. doi: 10.1103/PhysRevD.82.123512

62. Feng WZ, Mazumdar A, Nath P. Baryogenesis from dark matter in an inflationary universe. Phys Rev. (2013) D88:036014. doi: 10.1103/PhysRevD.88.036014

63. Feng WZ, Nath P, Peim G. Cosmic coincidence and asymmetric dark matter in a stueckelberg extension. Phys Rev. (2012) D85:115016. doi: 10.1103/PhysRevD.85.115016

64. Blennow M, Dasgupta B, Fernandez-Martinez E, Rius N. Aidnogenesis via leptogenesis and dark sphalerons. JHEP (2011) 1103:014. doi: 10.1007/JHEP03(2011)014

65. Barr SM, Chen HY. Cogeneration of dark matter and baryons by nonstandard-model sphalerons in unified models. JHEP (2013) 1310:129. doi: 10.1007/JHEP10(2013)129

66. Buckley MR, Randall L. Xogenesis. JHEP (2011) 1109:009. doi: 10.1007/JHEP09(2011)009

67. Gu PH, Lindner M, Sarkar U, Zhang X. WIMP dark matter and baryogenesis. Phys Rev. (2011) D83:055008. 10.1103/PhysRevD.83.055008

68. Allahverdi R, Dutta B, Sinha K. Cladogenesis: baryon-dark matter coincidence from branchings in moduli decay. Phys Rev. (2011) D83:083502. doi: 10.1103/PhysRevD.83.083502

69. Kohri K, Mazumdar A, Sahu N, Stephens P. Probing unified origin of dark matter and baryon asymmetry at PAMELA/Fermi. Phys Rev. (2009) D80:061302. doi: 10.1103/PhysRevD.80.061302 
70. Cheung C, Zhang Y. Electroweak cogenesis. JHEP (2013) 1309:002. doi: 10.1007/JHEP09(2013)002

Conflict of Interest Statement: The authors declare that the research was conducted in the absence of any commercial or financial relationships that could be construed as a potential conflict of interest.

Received: 09 October 2013; accepted: 17 December 2013; published online: 24 January 2014.
Citation: Boucenna SM and Morisi S (2014) Theories relating baryon asymmetry and dark matter. Front. Physics 1:33. doi: 10.3389/fphy.2013.00033

This article was submitted to High-Energy and Astroparticle Physics, a section of the journal Frontiers in Physics.

Copyright (c) 2014 Boucenna and Morisi. This is an open-access article distributed under the terms of the Creative Commons Attribution License (CC BY). The use, distribution or reproduction in other forums is permitted, provided the original author(s) or licensor are credited and that the original publication in this journal is cited, in accordance with accepted academic practice. No use, distribution or reproduction is permitted which does not comply with these terms. 